\title{
USRP Implementation of Spectrum Sensing OFDM-Based Cognitive Radio Networks Using Energy Ratio Algorithm
}

\author{
Sohila Abdalla Mohamed ${ }^{1, *}$, Abdelrahim Ate ${ }^{2}$ \\ 1,2 Graduate college, Alneelain University, Khartoum, Sudan \\ 2 School of Mechanical and Electrical Engineering, Dezhou University, China \\ * Corresponding author: Sohila Abdalla Mohamed (sohilafagiri@gmail.com). \\ Article history: Received 17 July 2021, Received in revised form 04 October 2021, Accepted 14 October 2021 \\ Digital Object Identifier (doi): 10.52981/fjes.v11i1.803
}

\begin{abstract}
This paper explains the Universal Software Radio Peripheral (USRP) Experiment results of Spectrum Sensing Algorithms based on the Energy Ration Algorithm for Cognitive Radio Networks which is latterly suggested in Spectrum observation for OFDM-Based Cognitive Radio Networks by using Energy Ratio Algorithm. This is completed through detecting the variance in the strength of the signal during a variety of confined OFDM subcarriers are used to ensure that the availability of the essential user is facilely discovered. Extensive experiments are performed, in particular, the effects of Signal to Noise Ratio (SNR). This paper observed that the experimental results gave lower detection performance compared to the simulation results. That's due to existence of other systems which operate on same frequency band of $2.4 \mathrm{GHz}$.
\end{abstract}

Keywords: Spectrum Sensing, Cognitive Radio, OFDM, USRP.

\section{INTRODUCTION}

Over a long time ago, conventional methods to spectrum administration are challenged by modern experiences in the particular utilize of spectrum [1]. In many countries, spectrum at frequencies lower down $3 \mathrm{GHz}$ was completely apportioned to particular utilizations. For instance, the Federal Communication Commission's (FCC) frequency allotment scheme point out numerous allocations over whole of the frequency bands [2]. Consequently, within the present regulative framing, spectrum may be an infrequent resource, in a minimum at the frequencies below $3 \mathrm{GHz}$, that are especially valuable because of their suitable spread Properties. On the other side, actual assessments that got it at the BWRC shows out low uses in between 0 to $2.5 \mathrm{GHz}$ bands [3]. These estimations put doubt on the efficiency of the current regulatory system.

As the estimations appear plainly, that who are allocated frequency bands by the administrative body (Essential Clients) are not utilizing it at the most time. At the same time, other clients want to utilize spectrum but do not have a prerogative to use the identical frequencies.

Wherefore, the way to increasing spectrum efficacy is to allow these others, Secondary Users permit access to frequency bands previously allocated to Essential Users whilst these are not utilizing it. Opportunistic Spectrum Sharing is one of the techniques could accomplish this sharing. In accordance with the opportunistic sharing system, Secondary Users are permit to work in same specific frequency bands without the approval of the Primary Users of these frequency bands, as long as they are not intervened with the Essential Client [4]. 
The FCC was authorized this type of sharing in the $5 \mathrm{GHz}$ band and also consider permitting it in the TV broadcasting bands [5]. To execute opportunistic sharing, the Cognitive Radios are innovation which will be utilized. Cognitive Radios have the ability to sense the spectrum range to consider if it's being used by the Essential Client.

And so on, this sensing process may be difficult because of deteriorated wireless channel, which has motivated concerns from Primary users of the spectrum [4]. OFDM stands for orthogonal frequency division multiplexing, and it is a type of multi-carrier modulation mechanism which used in much systems of wireless and assured as a trustworthy and efficient mode of transmission. For these reasons, OFDM is most favored for cognitive networks and it has already been used into the existing IEEE 802.22 cognitive standard. in other side, traditional OFDM there are difficulties in systems that conventional spectrum sensing techniques put their procedure on the time-domain samples that have been received to detect a specific characteristic to the essential user [6]. Factually, the signal structure of the minor user can help in the spectrum sensing. while the secondary client uses OFDM to set as the physical transmission technique, appoints approach based on a frequency space and that to sense the spectrum range in all the reception as long as the transmitter of the secondary user gives an extra advantage to the normal signal of OFDM [7]. Stimulated by the request for spectrum sensing in the way of OFDM modulated signals. in the paper, an experiment, styling of spectrum sensing algorithm based on Energy Ratio Algorithm for OFDM-Based Cognitive Radio Networks which is recently proposed in [7-9].

\section{METHODOLOGY}

\section{A. SYSTEM ANDBACKGROUND} MODEL

In generally the received signal given by $x(t)=s$ $(\mathrm{t})+\mathrm{n}(\mathrm{t})$, where:

$\mathrm{s}(\mathrm{t})=$ the signal of primary user

$\mathrm{n}(\mathrm{t})=$ white noise assumed that: $\mathrm{fc}=$ frequency band, $\mathrm{W}=$ bandwidth, $\mathrm{fs}=$ sampled

Where $W$, Ts and $f s=1$ / fs as sampling period.

So determined $x(n)=x(n T s), s(n)=s(n T s)$ and $n$ (n) $=$ n (nTs).

If there is no signal, $\mathrm{H} 0$ is utilized, and if there is, $\mathrm{H} 1$ is used, these are the two assumptions that are employed [10].

$$
\begin{array}{ll}
H_{0}: & x(n)=w(n), \quad n=0, \ldots, N-1 \\
H_{1}: & x(n)=s(n)+w(n) n=0, \ldots, N-1
\end{array}
$$

Where $x(n)$ is an independent identically distributed (IID). $\mathrm{W}(\mathrm{n})$ is circularly symmetric complex Gaussian (CSCG) with zero mean and variance $\sigma^{2} \mathrm{I}$, with $\mathrm{I}$ is the identity matrix. Deem that an OFDM with $\mathrm{N}$ subcarriers; the complex data are modulated into the $\mathrm{N}$ subcarriers over inverse discrete Fourier transform (IDFT). By the supposition that $\mathrm{L}$ is the length of the Cyclic Prefix (CP), Let $h(l)$ refer to the impulse response of the channel, On the receiver side, the received sampled data $x(n)$ can be written as [8]

$$
P_{D}=\operatorname{Pr} o b[X>\gamma]=1-I \frac{\left(\sigma \frac{2}{v} \gamma / \sigma \frac{2}{u}\right)}{1+\left(\sigma \frac{2}{v} \gamma / \sigma \frac{2}{u}\right)}(N, N)
$$

The spectrum sensing system in this paper is designed as in Fig1.[6].

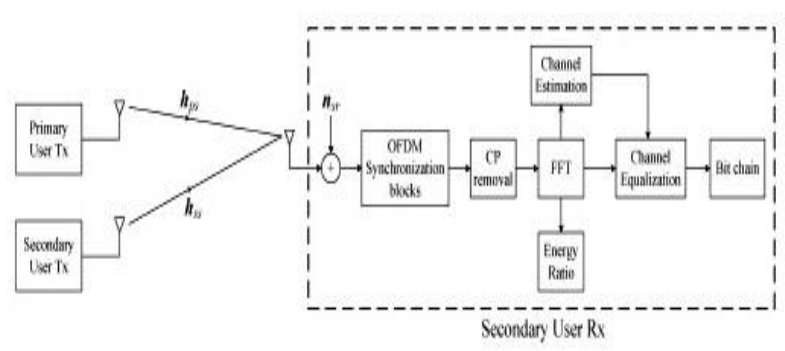

Fig. 1 SISO system communication model that considers both primary and secondary user channels [7].

At the time-frequency net of the OFDM frame and prior the IDFT, a number of tones are confined for the spectrum sensing purposes. The proposed OFDM frame is shown in Fig. 2 [7]. 
Suppose that the signal to be discovered does not have any recognized structure that could be exploited. As a result, a zero mean circularly symmetric complex Gaussian distribution is used to model the reserved tone sequence [10].

The goal of this analysis is to determine the receiver operating characteristics (ROC) exemplified by the probability of detection (PD) and probability of false alarm (PFA). The classical NP criterion was developed to acquire the ROC, in which the discovery likelihood is raised while the false alert prospect is kept constant. When the probability of detection for the energy ratio algorithm is calculated by (2), The limit can be gained by applying a constant PFA.

$$
x[n]=e^{j(2 \pi f \Delta n / L N)} \cdot \frac{1}{N} x(n-l)+\omega(n)
$$

The receiver calculates the energy ratio of reserved tones and if it crosses a specific limit, the secondary user supposed that the power on their served tones has changed, possibly as a result of the primary user's presence. If not, the transmission can be continued by the secondary user.

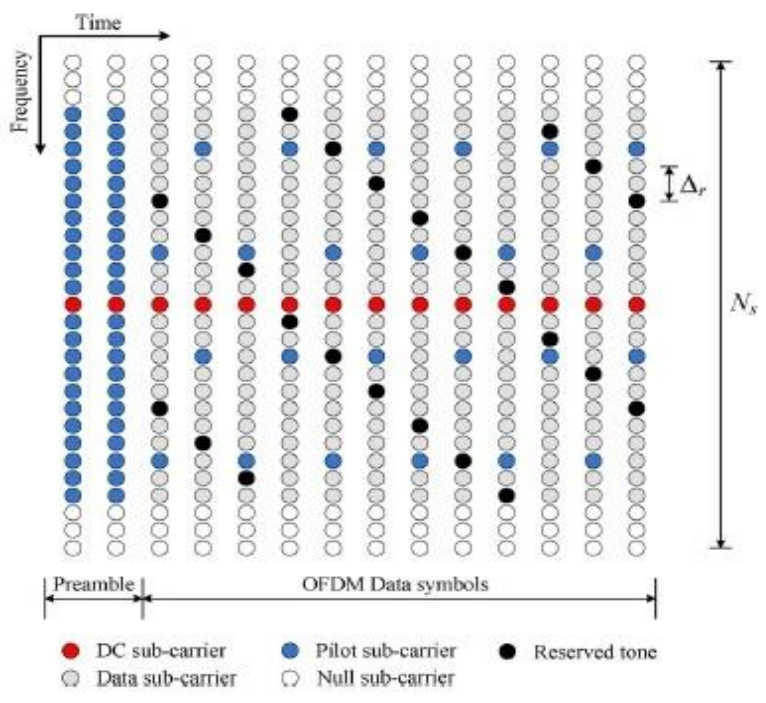

Fig. 2. Time-frequency allotment for one OFDM frame to scout various sub-carrier formats [6].

Actually, if there no exists of the primary user in the band, the energy of each window consists solely of the strength of undesired signals, included noise, if the spectrum sensing algorithm determines that the primary user is not active, then properly the energy ratio algorithm has been tuned to sense the primary user's existence. Because the strength of the undesired signals does not give alteration significantly over time, the ratio will be extremely close to unity if $\mathrm{N}$ is large enough. Meantime, the first window will just preserve the undesired signals without the primary user confusion. When compared to one, the ratio of the two energies will result in substantially greater values [7].

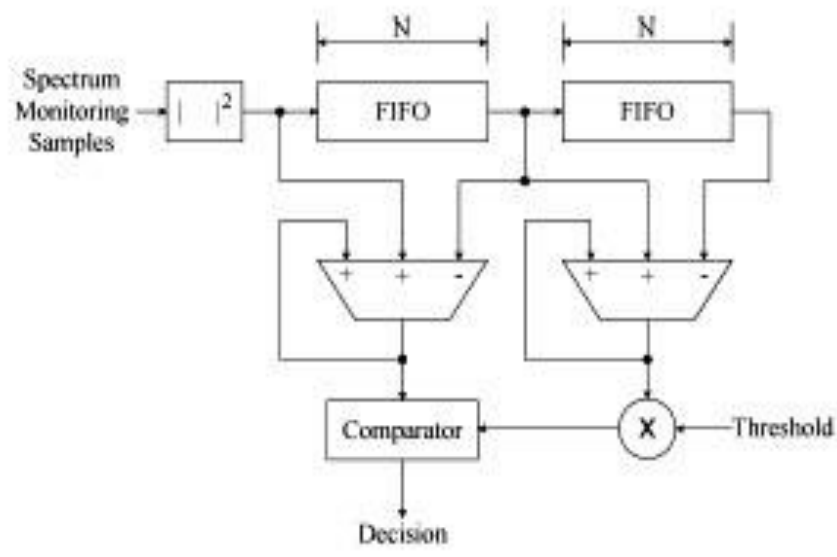

Fig. 3. The energy ratio algorithm's proposed architecture [6 7]

\section{B. Experimental Parameters}

The artifice is a sum of $\mathrm{N}=1024$ sub-carriers, with 224 serving as guard bands on each extremity of the signal band. Over the course of the 800 sub-carriers, there are 32 pilot subcarriers and four reserved tones. The sampling frequency is $16 \mathrm{MHz}$, and the cyclic prefix $\mathrm{L}=64$ samples long. The subcarrier spacing becomes $\mathrm{f}=15.625 \mathrm{KHz}$, which is big enough to ignore phase noise deformation and time domain windowing impact. Two sequential practices symbols, pawned tone spacing of $\mathrm{r}=2$ and 256 OFDM data symbols, are all included in the frame unless otherwise stated. 


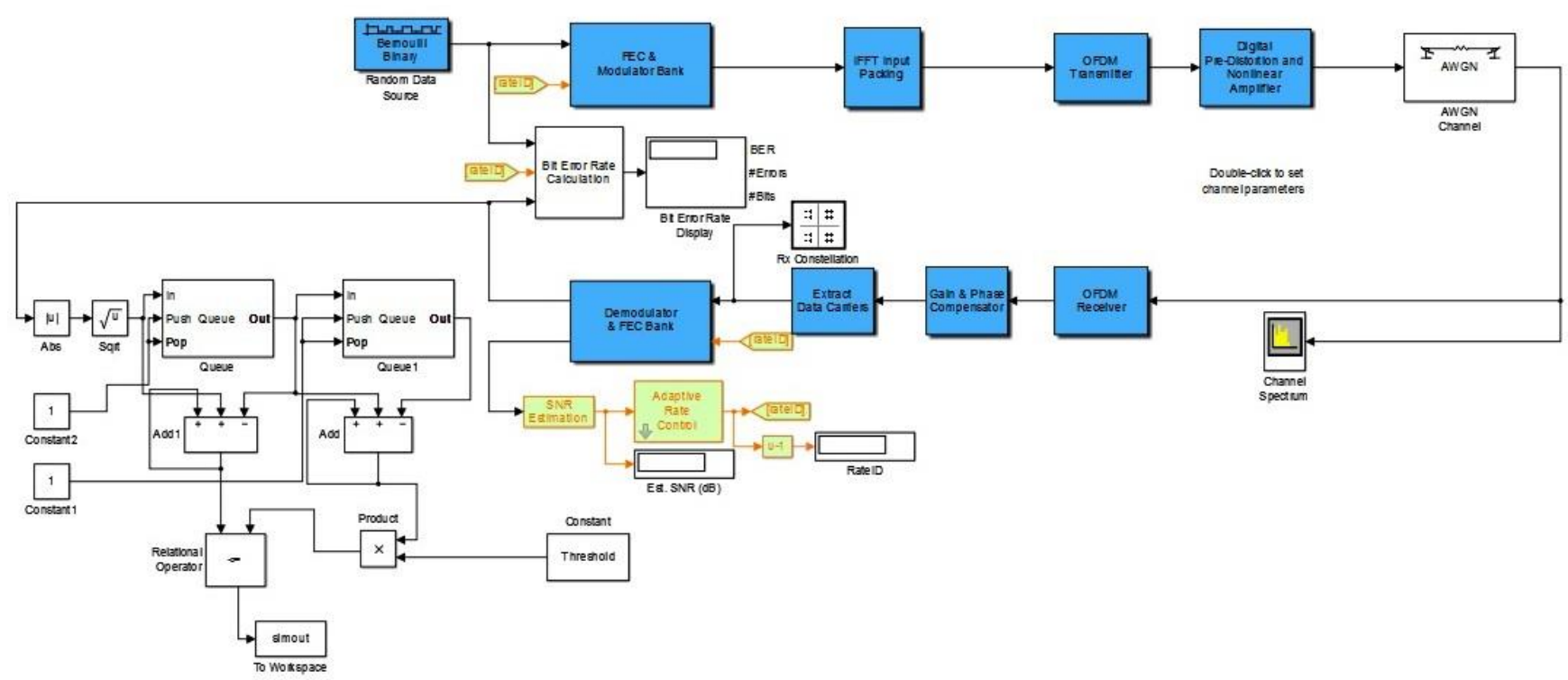

Fig. 4. The schematic of secondary user implementation of OFDM modulator (top) and demodulator (bottom) under AWGN channel.

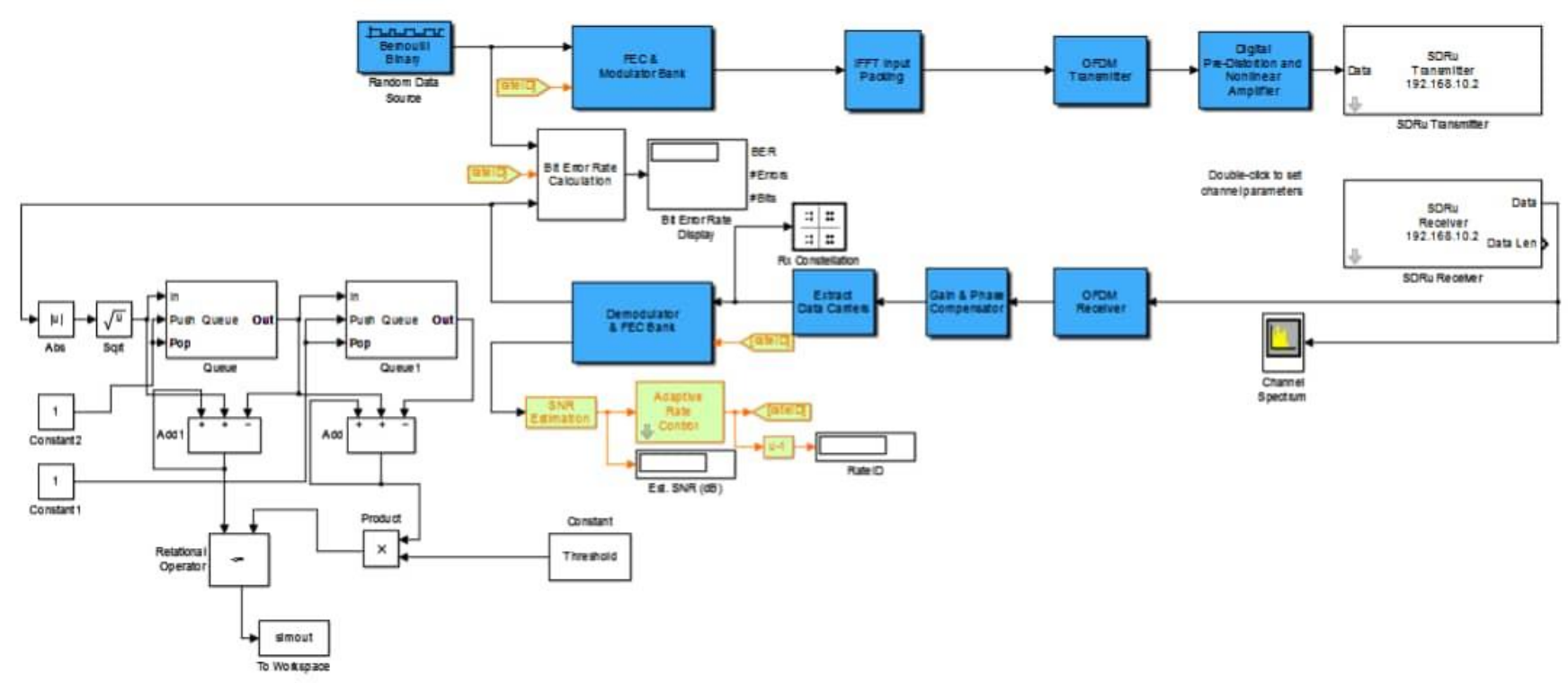

Fig. 5. The schematic of secondary user implementation of OFDM modulator (top) and demodulator (bottom) using USRP real time RF frequency centered at $2.4 \mathrm{GHz}$.

\section{RESULTS AND DISCUTIONS}

The USRP is a device that can generate a software radio with any computer that has a gigabit Ethernet interface controller, an SD card port, and a MIMO expansion slot on the front end, as well as eight LED indications. The driver for the USRP mother board and RF transceiver is stored on the SD card. USRP2 requires $5 \mathrm{~V}$ DC and $6 \mathrm{~A}$ to operate [11]. The aim behind using USRP is to execute all signal processing duties on the host computer, such as modulation and demodulation, and filtration. The USRP performs all generalpurpose activities such as interpolation, decimation, digital up conversion, and down conversion [12]. Fig. 6 shows the experimental environment using an USRP Architecture. The sensing test lab consists of one Ettus Research USRP (shown in Fig.6) coupled to a host PC running a MATLAB/Simulink Software-based processing software. The system model in Fig.5 was created with the Signal Processing Toolbox integrated with Simulink and the comprehensive Communication System Toolbox. The test bed is 
transformed into a complete RF transceiver system when the RF front-end XCVR2450 daughterboard is interfaced with USRP which supports a frequency of $2.4 \mathrm{GHz}$. To get a twoway high-bandwidth communication, a VERT2450 Vertical Antenna with a gain of $3 \mathrm{dBi}$ is used.

The test bed is designed to run at a frequency of $2.4-2.5 \mathrm{GHz}$, which is a license-free band that corresponding to the channel of real-world radios. The data were transmitted via the USRP sender, The receiver detects the frequency range between 2.4 and $2.5 \mathrm{GHz}$ and corrects the offset frequency automatically.

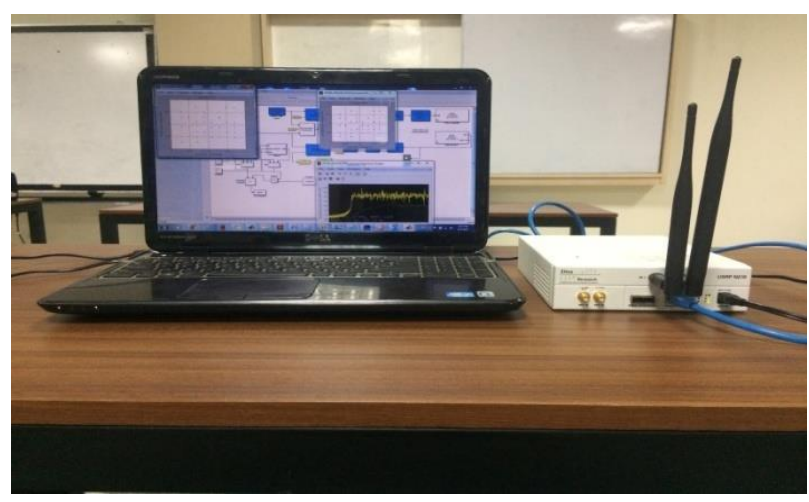

Fig. 6. The experimental environment

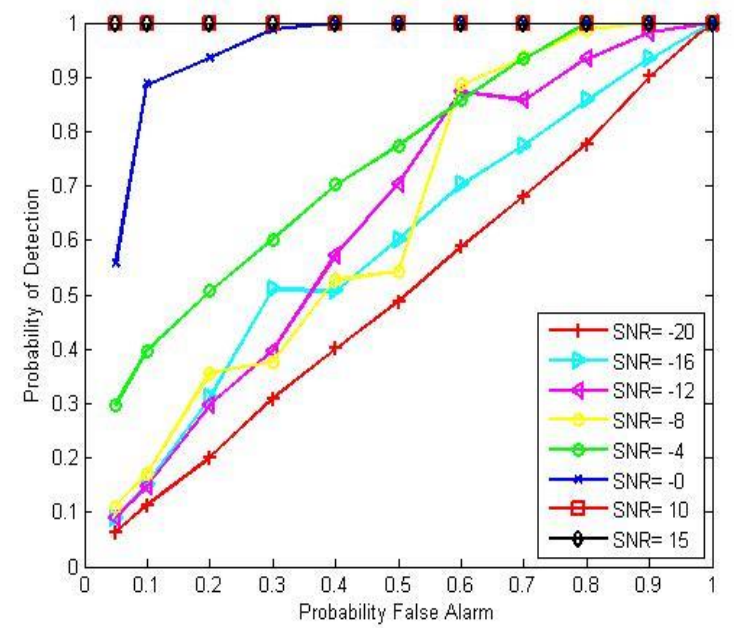

Fig. 7. Receiver operating characteristics simulation for various SNR values with perfect synchronization and AWGN channel

\section{CONCLUTION}

This paper deals with description of experimental of energy ration spectrum sensing algorithm based on USRP modules available from ETTUS research. The aim of this paper is to test the algorithm for OFDM-based cognitive radio mobile networks. An extensive hardware and software environment have been integrated to optimize the experimentation process. Here were able to detect other systems that operate on the $2.4 \mathrm{GHz}$ band using this arrangement because the module can measure the spectrum of the 2.4-2.5 $\mathrm{GHz}$ band.

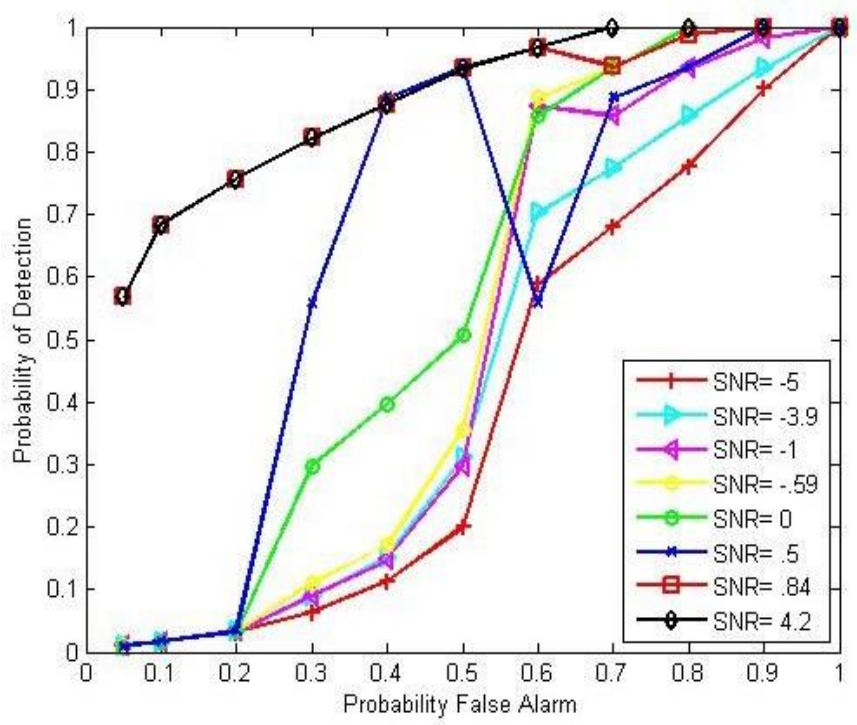

Fig. 8. Receiver operational characteristics experimental results for several SNR values estimated for $2.4 \mathrm{GHz}$ USRP broadcast and reception in real time

Furthermore, this prototype provides a triedand-true platform for the study and implementation of cognitive radio approaches. As demonstrated in Figure. 7 and 8, examination the influence of SNR on the PD of the algorithm of [6] for various SNR values. The PD of the algorithm in this study grows as the SNR increases, as can be seen in this figure. Otherwise, observed that the experimental channel differs from the AWGN channel, which is to be expected [13-15]. This is owing to the fact that the transmission and reception frequencies are both $2.4 \mathrm{GHz}$, which causes a lot of interference with other radio communication systems that use the same frequency band [16].

\section{REFERENCES}

[1]. Chtaut, R., \& Akl, R. . Massive MIMO Systems for 5G and beyond Networks-Overview, Recent Trends, Challenges, and Future Research Direction. Sensors, 20(10), 2753, 2020.

[2]. Harold Furchtgott-Roth, "Unlicensed operation in the tv broadcast bands and additional spectrum for unlicensed devices below $900 \mathrm{mhz}$ in the $3 \mathrm{ghz}$ band, in Federal 
Communications Commission", NOTICE OF PROPOSED RULE MAKING, pp. 04-186, 2004.

[3]. D. Cabric, S. M. Mishra, D. Willkomm, R. W. Brodersen, and A. Wolisz, "A cognitive radio approach for usage of virtual unlicensed spectrum", in 14th IST Mobile Wireless Communications Summit 2005, Dresden, Germany, 2005.

[4]. Kodheli, O., Lagunas, E., Maturo, N., Sharma, S. K., Shankar, B., Montoya, J. F. M., ... Goussetis, G. Satellite Communications in the New Space Era: A Survey and Future Challenges. IEEE Communications Surveys \& Tutorials, pp. 1-1, 2020.

[5]. T. Weiss and F. Jondral, "Spectrum pooling: An innovative strategy for the enhancement of spectrum efficiency", IEEE Communications Magazine. 42(3), pp. 8-14, 2004.

[6]. J.Mitola, "Cognitive radio: an integrated agent architecture for software defined radio," Ph.D. thesis, Royal Institute of Technology, Sweden, 2000.

[7]. Steven M. Kay, "Fundamentals of Statistical Signal Processing: Detection Theory", Vol.2, PrenticeHall, 1993.

[8]. Abdelmohsen Ali and WalaaHamouda, "Spectrum Monitoring Using Energy Ratio Algorithm for OFDM-Based Cognitive Radio Networks". IEEE Transactions on Wireless Communications 14(4), pp. 2257-2268, 2015.

[9]. Hou-Shin Chen and David G. Daut, "Sensing for OFDM Systems Employing Pilot Tones", IEEE TRANSACTIONS ON WIRELESS COMMUNICATIONS.8(12), pp. 5862-5870, 2009.

[10]. Wen-Long Chin, Chun-Wei Kao, and Yi Qian, "Spectrum Sensing of OFDM Signals Over Multipath Fading Channels and Practical Considerations for Cognitive Radios", IEEE Sensors Journal. 16(8), pp. 2349-2360, 2016.

[11]. Tadilo Endeshaw Bogale and Luc Vandendorpe, “USRP Implementation of Max-Min SNR Signal Energy based Spectrum Sensing Algorithms for Cognitive Radio Networks, Cognitive Radio and Networks Symposium", .IEEE International Conference on Communications (ICC), 2014.

[12]. Mody, A., Chouinard, G., Shellhammer, S. J., Ghosh, M., \& Cavalcanti, D, "Cognitive Wireless Regional Area Network Standard", Opportunistic Spectrum Sharing and White Space Access, pp, 551-603, 2015.

[13]. [Garg, V. K, "Fourth Generation Systems and New Wireless Technologies", Wireless Communications \& Networking, pp. 23-22, 2007

[14]. [Yu, S., Liu, J., Wang, J., \& Ullah, I. "Adaptive DoubleThreshold Cooperative Spectrum Sensing Algorithm Based on History Energy Detection", Wireless Communications and Mobile Computing, pp. 1-12, 2020.

[15]. Bogale, T. E., \& Vandendorpe, L, “USRP implementation of Max-Min SNR signal energy-based Spectrum Sensing Algorithms for Cognitive Radio Networks", IEEE International Conference on Communications (ICC), 2014.

[16]. Lei, Z., Yang, P., \& Zheng, L, "Detection and Frequency Estimation of Frequency Hopping Spread Spectrum Signals Based on Channelized Modulated Wideband Converters.", Electronics, 7(9), pp. 170, 2018. 